\title{
Measurement and removal of cladding light in high power fiber systems
}

Till Walbaum, Andreas Liem, Thomas Schreiber, Ramona Eberhardt, Andreas Tünnermann

Till Walbaum, Andreas Liem, Thomas Schreiber, Ramona Eberhardt, Andreas Tünnermann, "Measurement and removal of cladding light in high power fiber systems," Proc. SPIE 10513, Components and Packaging for Laser Systems IV, 105130U (20 February 2018); doi: 10.1117/12.2288266

SPIE. Event: SPIE LASE, 2018, San Francisco, California, United States 


\title{
Measurement and removal of cladding light in high power fiber systems
}

\author{
Till Walbaum*a, Andreas Liem ${ }^{\mathrm{a}}$, Thomas Schreiber ${ }^{\mathrm{a}}$, Ramona Eberhardt ${ }^{\mathrm{a}}$, Andreas Tünnermann ${ }^{\mathrm{a}, \mathrm{b}}$

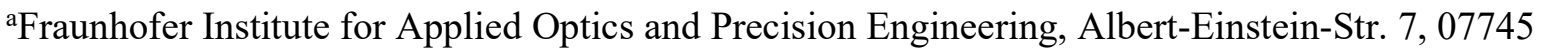 \\ Jena, Germany; \\ ' Institute of Applied Physics, Abbe Center of Photonics, Friedrich-Schiller-University Jena, Albert- \\ Einstein-Str. 15, 07745 Jena, Germany \\ *corresponding author: till.walbaum@iof.fraunhofer.de
}

\begin{abstract}
The amount of cladding light is important to ensure longevity of high power fiber components. However, it is usually measured either by adding a cladding light stripper (and thus permanently modifying the fiber) or by using a pinhole to only transmit the core light (ignoring that there may be cladding mode content in the core area). We present a novel noninvasive method to measure the cladding light content in double-clad fibers based on extrapolation from a cladding region of constant average intensity. The method can be extended to general multi-layer radially symmetric fibers, e.g. to evaluate light content in refractive index pedestal structures.

To effectively remove cladding light in high power systems, cladding light strippers are used. We show that the stripping efficiency can be significantly improved by bending the fiber in such a device and present respective experimental data. Measurements were performed with respect to the numerical aperture as well, showing the dependency of the CLS efficiency on the NA of the cladding light and implying that efficiency data cannot reliably be given for a certain fiber in general without regard to the properties of the guided light.
\end{abstract}

Keywords: double clad fiber, cladding light, cladding light stripper, fiber components, fiber laser characterization, high power fiber laser

\section{INTRODUCTION}

To allow for brightness enhancement in fiber laser systems, double clad fibers are commonly used in high power setups. The amplifying fibers in such systems usually comprise an actively doped core of small diameter and numerical aperture, allowing for low modal content and thus high brightness light guidance. To convert low brightness pump light, the latter is coupled into a larger pump core with a significantly higher numerical aperture (usually 0.22 or 0.46 ) surrounding the actively doped signal core. Due to the spatial overlap between pump core (aka cladding) and signal core modes, light from the pump core is gradually absorbed in the signal core (the exact amount depending on the relation of the areas), enabling amplification of the signal light. However, pump light absorption is never $100 \%$, and signal light may also leak out of the signal core (e.g. due to defects or fiber bending) and propagate in the pump core. In high power systems, the amount of light in the pump core can easily go to the range of several Watts or even hundreds of Watts. Since subsequent fiber components or free-space optics are usually only designed for the signal light, this high power cladding light can cause considerable damage by absorption and scattering, either due to its localization in the pump core or due to its high NA (see Figure 1. It is thus essential to reliably evaluate the cladding light content and to remove it from the system.

Components and Packaging for Laser Systems IV, edited by Alexei L. Glebov, Paul O. Leisher, Proc. of SPIE

Vol. 10513, 105130U · (c) The Authors. Published under a Creative Commons Attribution

CC-BY 3.0 License · doi: 10.1117/12.221158

Proc. of SPIE Vol. $10513105130 U-1$ 


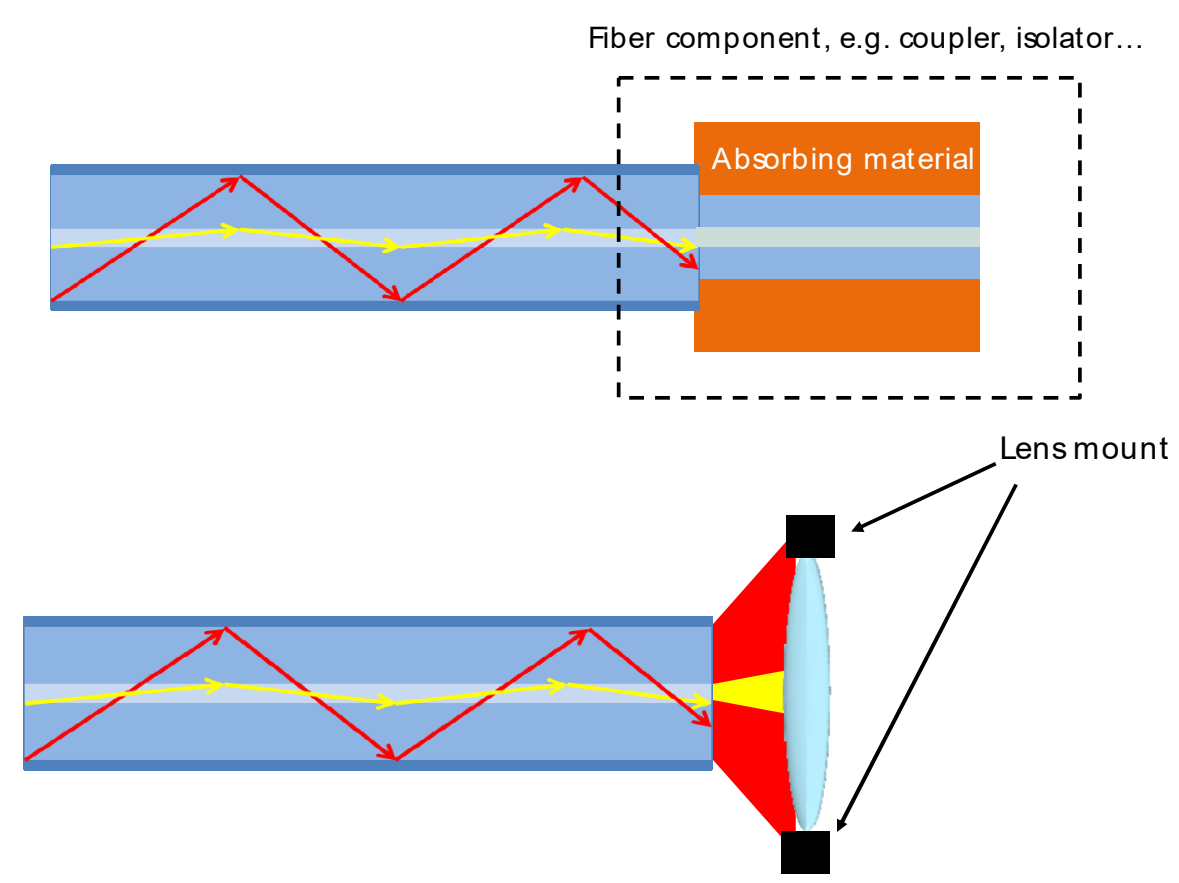

Figure 1: Potential problems caused by cladding light (red): absorption in non-low-index coating material due to location (top) or heating of optics' mounts due to high NA (bottom).

Within this paper, we will first introduce a method to measure the cladding light content of a double-clad fiber with reduced ambiguity that can be easily performed and is non-invasive. In the second part, we will provide new results on how to characterize and improve the efficiency of cladding light strippers that are essential parts of modern high power fiber systems.

\section{CLADDING LIGHT MEASUREMENT}

\subsection{Established methods and their limitations}

Commonly used methods for cladding light measurement can roughly be divided into far-field and near-field methods. While any measurement in the near field, i.e. the imaging plane of the fiber facet, basically characterizes the spatial distribution of the light, characterization in the far-field actually measures the numerical aperture power distribution and thus the spatial frequency distribution.

Measuring in the far field could be done by collimating a beam and cutting out the "central spot" (Figure 2, left). This, on the one hand, has the ambiguity that the clipping level is not well defined, as the shape of the angular distribution (and thus the far field beam profile) may vary. On the other hand, as indicated by the exemplary angular intensity distribution of the cladding light in the right part of Figure 2, cladding light can certainly have low-NA fractions and would thus be considered core light in such a measurement. 


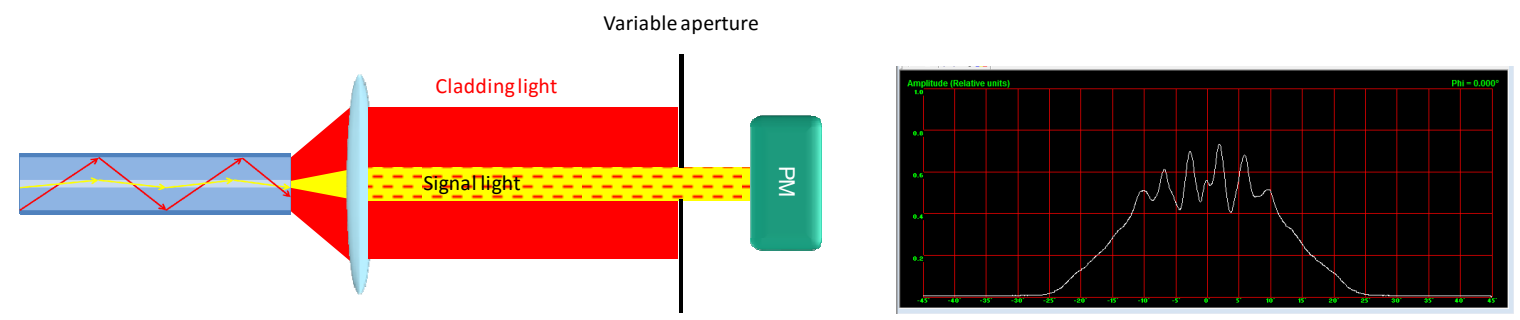

Figure 2: Measurement of the "core light" in the far field: Setup (left) and exemplary angular intensity distribution of cladding light (right).

Alternatively, one could measure in the near field by imaging the fiber facet to a camera and again cut out the core light with a variable aperture (left side, Figure 3), but again the clipping level is not defined. Furthermore, cladding light may also hold power content in the region of the core (which is, after all, why pump light is absorbed at all in a DCF), as indicated by the "cladding light only" intensity distribution on the right in Figure 3 (exemplary core indicated by red circle). Again, there are significant ambiguities.
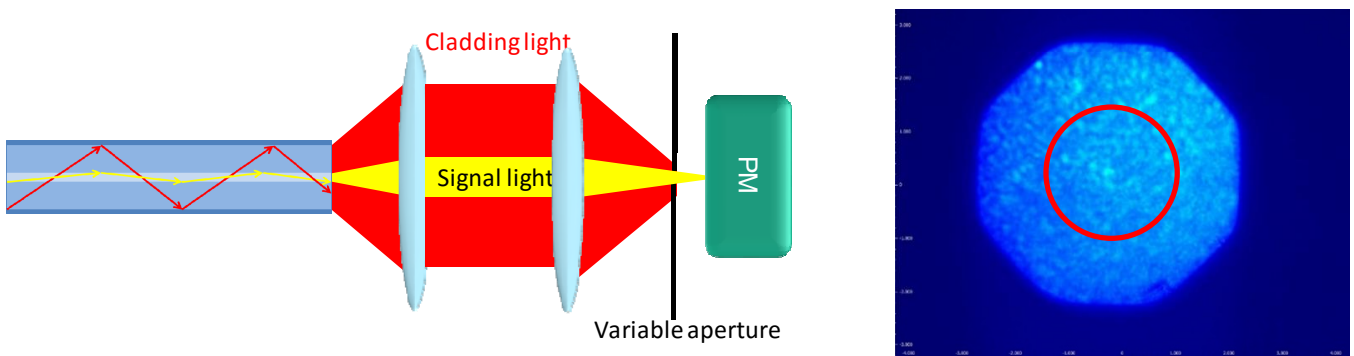

Figure 3: Measurement of "core light" in the near field (left) and near field intensity distribution of a DCF with no light coupled into the (signal) core. The red circle indicates a potential signal core, where the cladding light still holds intensity.

More rigorously, one could strip out the cladding light using a cladding light stripper (CLS) and compare the power before and after removing the cladding light. Unfortunately, this method is of course highly invasive and also does not take into account the (usually unknown) efficiency of the CLS, which also depends on the NA.

As an alternative, we propose what we call the constant-intensity-extrapolation (CIE) method: by assuming that the intensity is constant in the cladding region, we can either extrapolate the total power that this intensity would yield over the entire fiber region if the fiber geometry were known (CIE1). The difference between this estimate and the real measured total power yields the core power. On the other hand, if the fiber geometry is not well known, one can measure the power transmitted through a centered aperture of variable diameter in a setup similar to that shown in Figure 3 . By then plotting the measured power vs. the aperture area, we can identify the region of constant intensity representing the cladding region as a region of linear relation. Linearly extrapolating the power from this region to zero aperture area yields the power that would be measured if no cladding light was present, i.e. the core light only (CIE2).

\subsection{Numerical validation}

To validate the CIE evaluation methods, transverse intensity distributions consisting of super-Gaussian profiles with different diameters for core and cladding region were generate numerically. The cladding diameter was $80 \mu \mathrm{m}$ in all cases, and core radii of $20 \mu \mathrm{m}, 40 \mu \mathrm{m}$ and $60 \mu \mathrm{m}$ were taken into account. We compared the CIE1 and CIE2 methods to the "core only"-transmission measurement depicted in Figure 3 due to the obvious drawbacks of far field measurement (actually measuring the wrong magnitude) and CLS method (invasiveness). The power contents in core and cladding are given in Table 1. 
Table 1: Power in core and cladding for the cases used for numerical validation.

\begin{tabular}{|l|l|l|l|}
\hline Geometry & Core power & Cladding power & Cladding light content \\
\hline $20 / 80$ & $3831.2 \mathrm{~W}$ & $694 \mathrm{~W}$ & $13.34 \%$ \\
\hline $40 / 80$ & $17363 \mathrm{~W}$ & $6250.8 \mathrm{~W}$ & $26.47 \%$ \\
\hline $60 / 80$ & $39068 \mathrm{~W}$ & $6250.8 \mathrm{~W}$ & $13.79 \%$ \\
\hline
\end{tabular}

As an example for the "core only" method, the beam profile as seen through different aperture sizes is shown in Figure 4. Here, the problem of the lacking definition of the clipping level is clearly visible: one could argue anything transmitted through apertures between $32 \mu \mathrm{m}$ and $46 \mu \mathrm{m}$ radius as being "core light only". More precisely, Figure 5 gives the estimate of the cladding light content that one would make based on the chosen aperture diameter. Most probably, values would vary between $16 \%$ and $21 \%$ here.

\section{Open aperture}

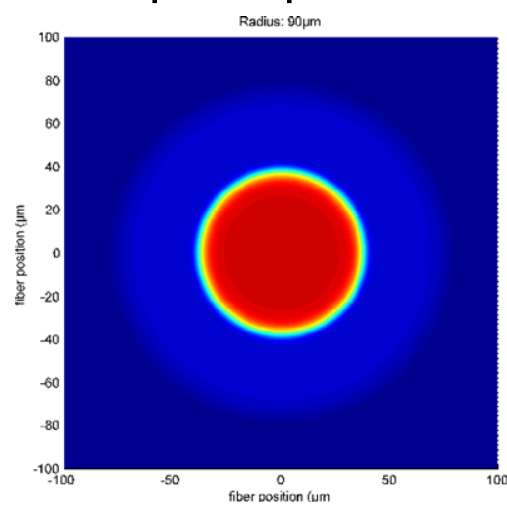

$32 \mu \mathrm{m}$ radius

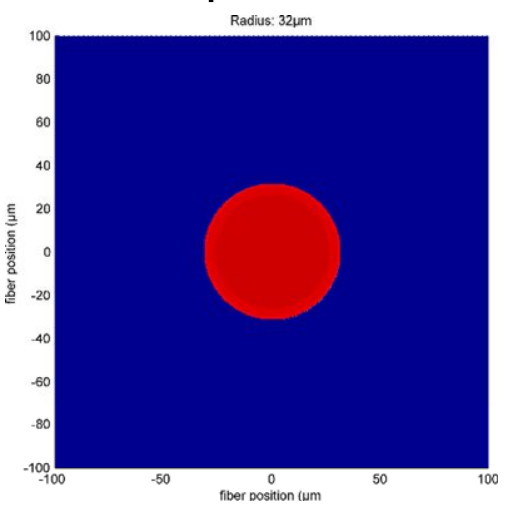

$46 \mu \mathrm{m}$ radius

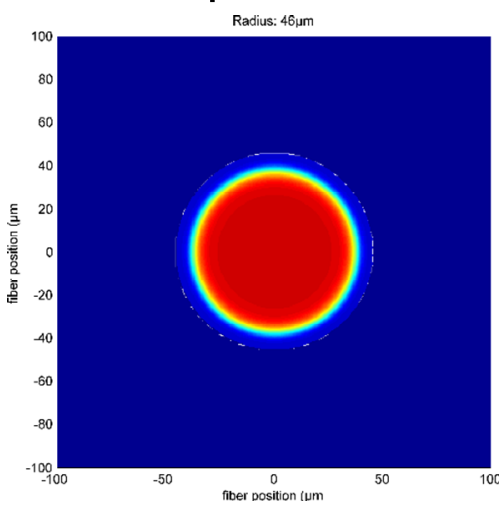

Figure 4: Beam profile with $\sim 40 \mu \mathrm{m}$ core radius (left) and transmitted beam profiles through apertures of $32 \mu \mathrm{m}$ (center) and $46 \mu \mathrm{m}$ (right) radius.

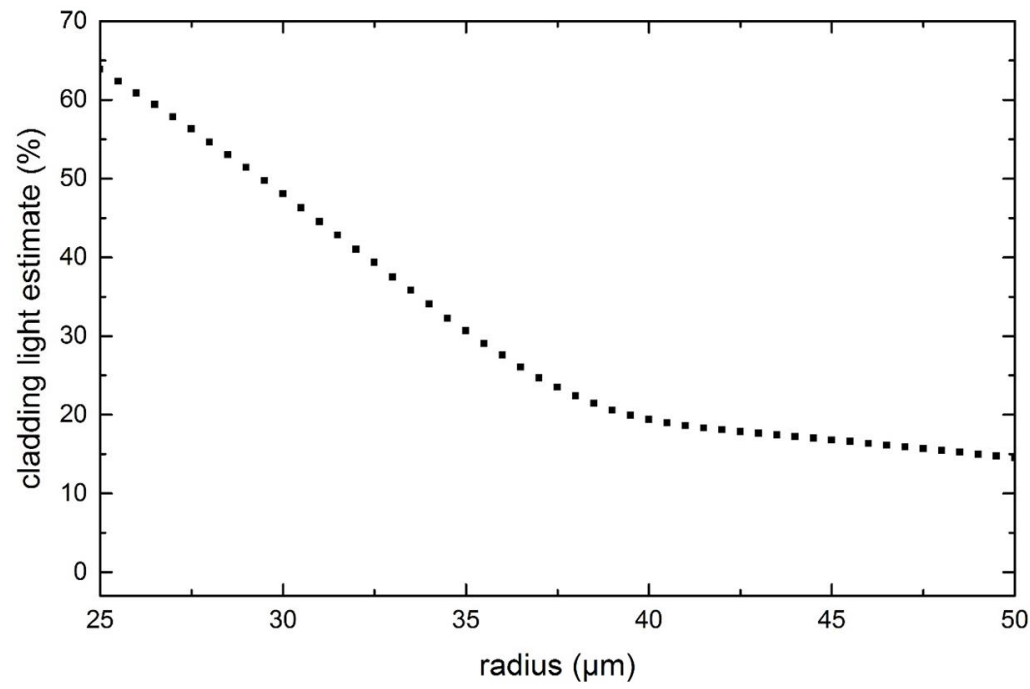

Figure 5: Estimated cladding light content in the $40 \mu \mathrm{m}$ core radius case, depending on the chosen aperture radius. 
In contrast, a more precise insight can be gained from the measurement of power vs. aperture area, as depicted in Figure 6 . The cladding region can easily be identified, on the one hand since we know where it should approximately be and on the other hand, since it is the outermost region of linear dependence between power and area. Of course, transmitted power is constant once the aperture area exceeds the fiber diameter. The cladding intensity, used for the CIE 1 method, is the slope of the linear fit. Using this value and the cladding radius of $80 \mu \mathrm{m}$, we would estimate around $7063.3 \mathrm{~W}$ cladding light, or $29.9 \%$. From the y-axis intersection of the fit, we can directly get the amount of light not in the cladding to be $17435.23 \mathrm{~W}$, corresponding to $26.16 \%$ cladding light.

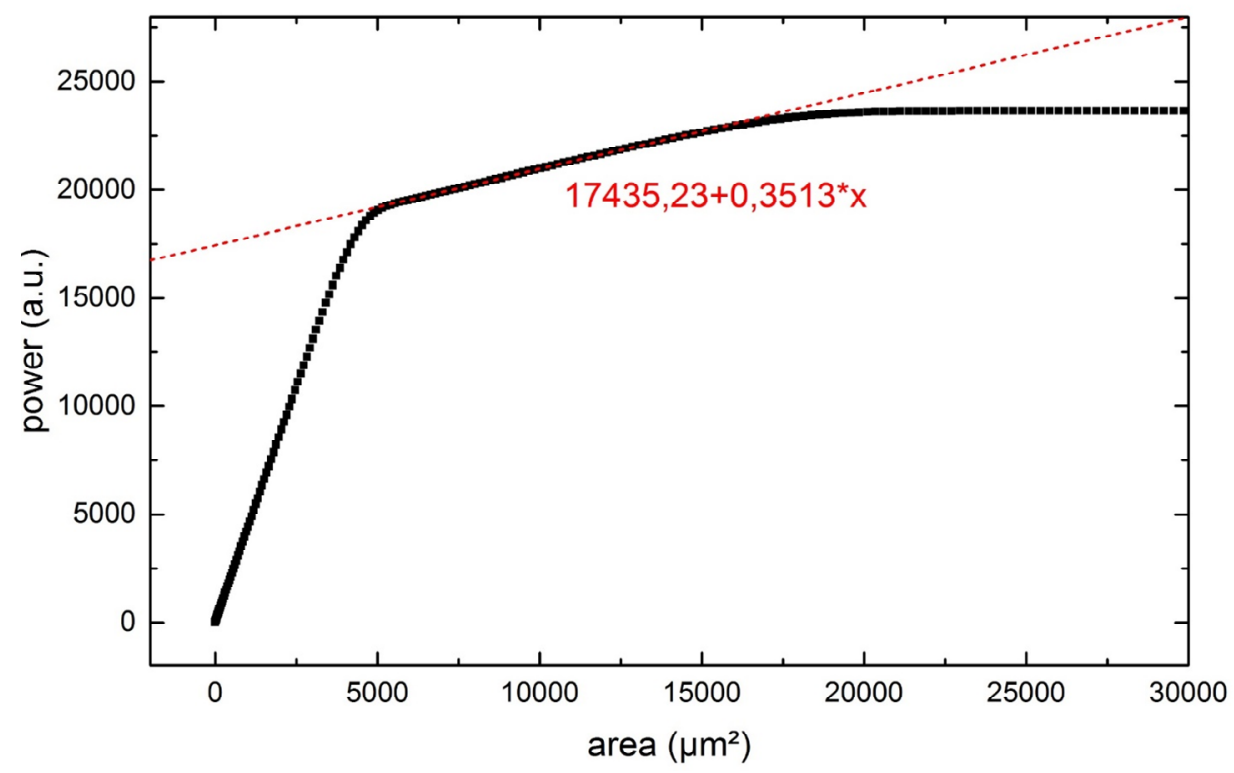

Figure 6: Transmitted power through aperture vs aperture area (black) and linear fit in the cladding region (red, identified by constant intensity and thus linear relation) for the $40 \mu \mathrm{m} / 80 \mu \mathrm{m}$ radius case.

For comparison, the results that the different methods would yield are shown in Table 2 for all three cases considered. It is clearly visible that the results of the "core only" measurement have the lowest precision and suffer most from higher core / cladding radius ratio. This is because the underlying cladding light content in the core region is not considered. The CIE2 method is the most precise for the lower ratios, but is surpassed by the CIE1 method for the highest ratio. This is because of the super-Gaussian shape of the considered beam profiles in the cladding, which is not flat close to the boundaries and thus violates the assumption of constant intensity there. In cases of small difference between core and cladding diameter, however, the fitting has to be done close to the cladding boundary, where the intensity still rises towards the center. This results in higher linear slopes from the fit than appropriate. Since the CIE1 method extrapolates outward (towards the assumption of "all fiber filled with cladding light") and the CIE2 method extrapolates inward (towards the assumption of "no cladding light"), an overestimated slope yields an estimate of too much core light (and too little cladding light) for CIE2 and too much cladding light for CIE1. However, both methods still beat the "core only" method significantly.

Table 2: Cladding light content estimates for different methods.

\begin{tabular}{|l|l|l|l|}
\hline $\begin{array}{l}\text { Core / cladding } \\
\text { radius }\end{array}$ & $\mathbf{2 0 ~ / 8 0}$ & $\mathbf{4 0 ~ / ~ 8 0}$ & $\mathbf{6 0 ~ / ~ 8 0 ~}$ \\
\hline $\begin{array}{l}\text { Real cladding } \\
\text { light content }\end{array}$ & $15.34 \%$ & $26.47 \%$ & $13.79 \%$ \\
\hline $\begin{array}{l}\text { "core only“ } \\
\text { estimate }\end{array}$ & $13 \%-15 \%$ & $16 \%-21 \%$ & $3.6 \%-10.7 \%$ \\
\hline CIE1 & $17.7 \%$ & $29.9 \%$ & $13.5 \%$ \\
\hline CIE2 & $15.32 \%$ & $26.16 \%$ & $12.3 \%$ \\
\hline
\end{tabular}




\section{EXPERIMENTAL VERIFICATION}

The experimental verification was performed using the setup depicted in Figure 7. Note that the restriction of needing to know the fiber geometry is impractical in real measurements, which is why CIE1 was not further investigated and only CIE2 was experimentally verified. The imaging distances could be easily aligned by imaging the octagonal fiber pump core onto the camera and by placing a calibration target with well-defined line spaces in the plane of the variable aperture (iris diaphragm). Note that a zero-aperture diaphragm was used to allow for investigation of small fiber cores. In addition, a high NA lens at the fiber output is required to allow for the imaging of the pump core, since it must capture the whole cladding light NA. The calibration target was used to calibrate the imaging scale, however, note that no calibration is necessary at all for the CIE2 to work as long as an aperture area can be defined for each power measurement. The power was measured using a low power semiconductor power meter.

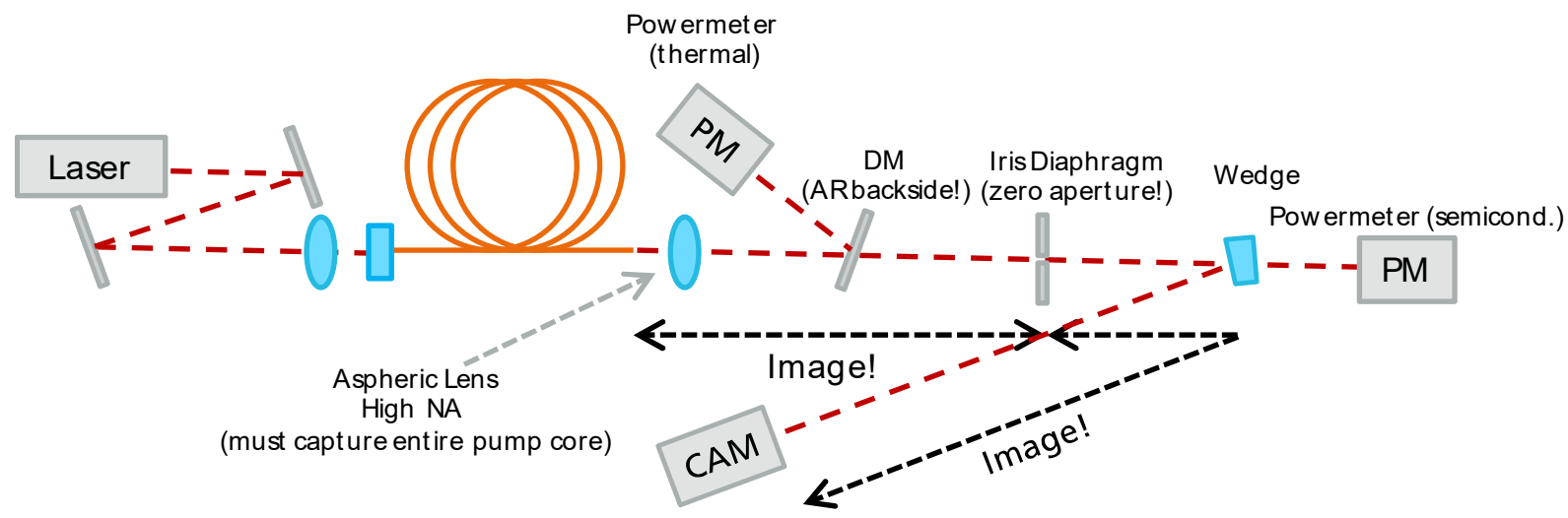

Figure 7: Experimental setup for cladding light measurement. PM: Powermeter; CAM: Camera; DM: Dichroic Mirror

With this setup, the cladding light content was measured for an in-house fiber containing significant scattering centers and exhibiting high cladding light power. For different aperture areas, the camera images are shown in Figure 8 . They allow for additional identification of the cladding area.

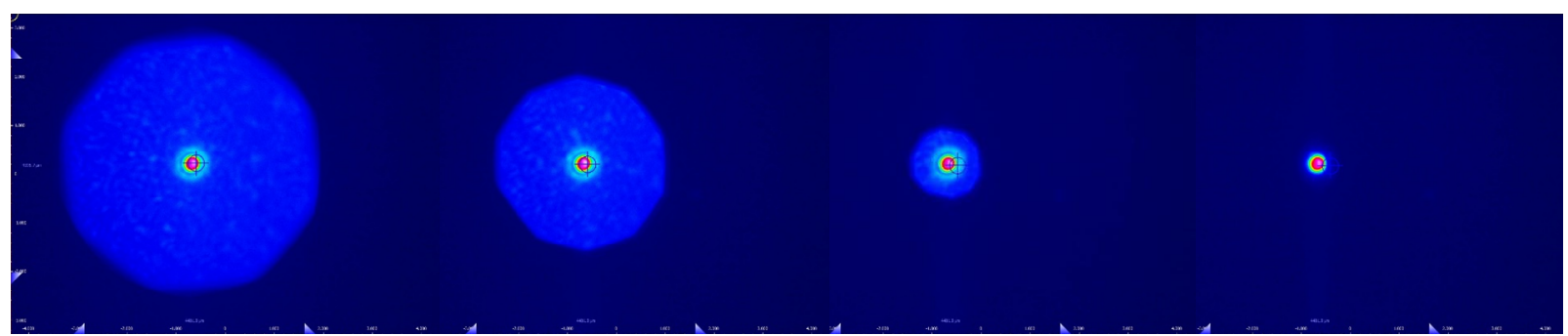

Figure 8: Camera image of the fiber facet for different stages of aperture closing. The octagonal pump core can be nicely seen with open aperture (left image).

The measured power is plotted over the aperture area in Figure 9. Even without the help of the camera images, a linear region can be easily identified (orange in the figure). A nonlinear relation between area and power can be observed in the core region (blue in the figure), while the power is constant for larger aperture diameter than that of the fiber cladding (green region). From the linear fit, a core light content of $15.56 \%$ (and thus $84.44 \%$ cladding light) could be determined. 


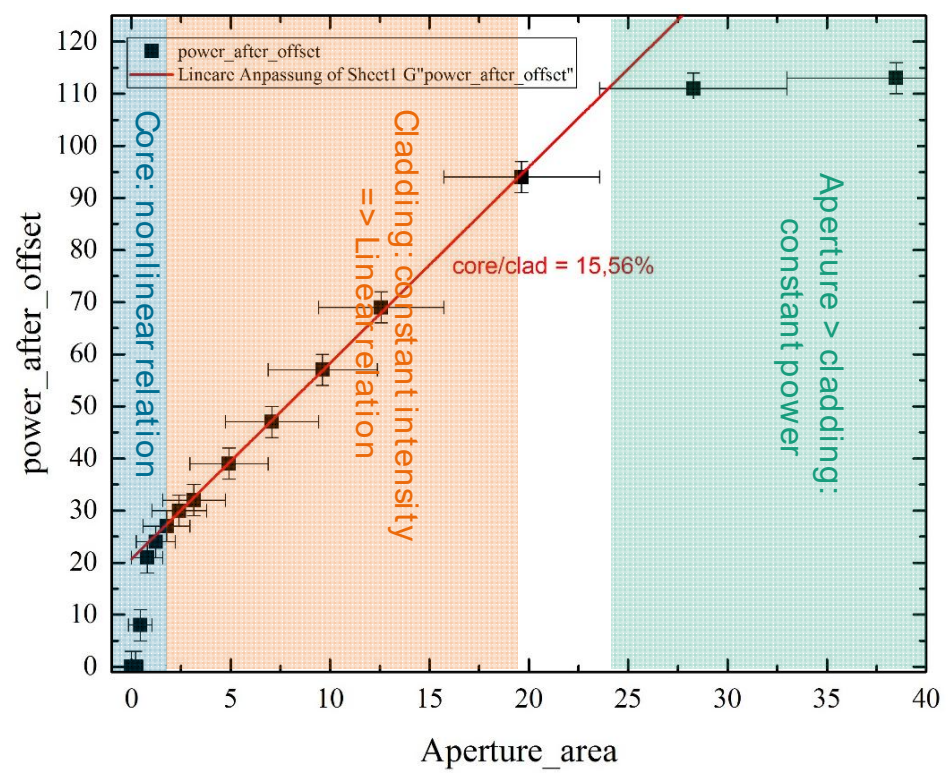

Figure 9: Measurement of cladding light content via CIE2 method. Constant intensity cladding region marked in orange

As an additional feature, the CIE2 method can be used to identify pedestal light content in triple clad fibers. These fibers have a pedestal of increased refractive index around a highly doped core, allowing for low signal core NA in spite of relatively high core refractive index. An example is shown in Figure 10, with the refractive index profile of the fiber shown as an inset. From the linear slope in the cladding region (orange dots), it can be determined that a total of $1.9331 \mathrm{~W}$ (out of $1.97 \mathrm{~W}$ output power in this measurement) was not in the cladding, yielding $1.9 \%$ cladding light content. The pedestal region is represented by the blue measurement points. Since power from cladding and pedestal region are present there, the fit tells us that $1.7342 \mathrm{~W}$ are neither in pedestal nor in cladding, representing a core power content of $88 \%$. Thus, the remaining $10.1 \%$ are guided in the pedestal.

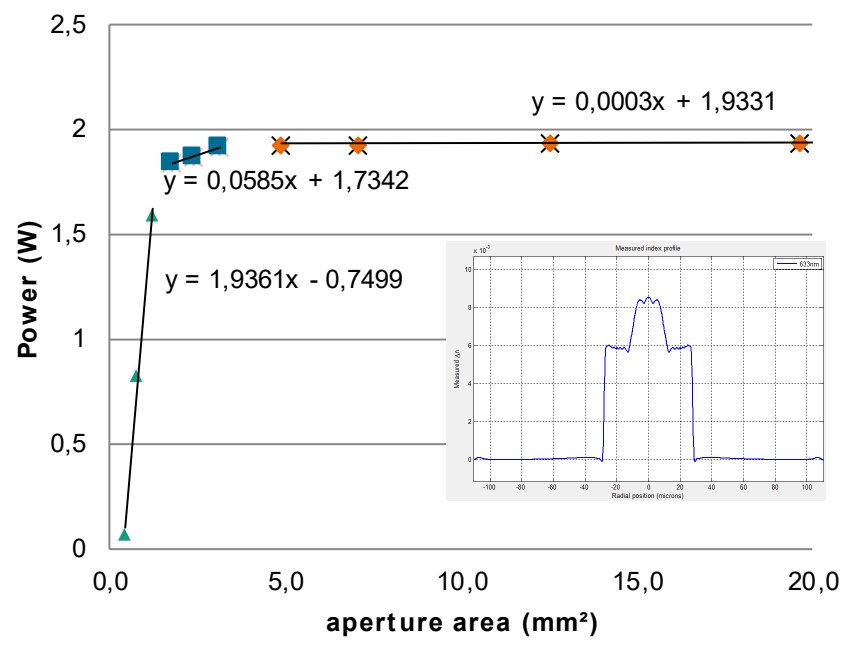

Figure 10: Measurement of cladding and pedestal light content for a triple clad fiber using CIE2 method. Inset: refractive index profile of the fiber. 


\section{CLADDING LIGHT REMOVAL}

Since cladding light can cause severe problems in high power fiber system, it usually needs to be removed. To achieve this goal, light guidance can be canceled by stripping off the low index acrylate coating of a double clad fiber and replacing it with high index material (e.g. UV adhesive) or structuring the fiber surface. The latter can be done by etching [1], engraving [2] or laser-based glass coating [3], for example.

A high dependence of the CLS efficiency on the NA of the guided light has been observed (e.g. [4]), suggesting that bending the fiber in the CLS and thus virtually increasing the NA could improve the efficiency. To investigate this, we constructed multiple cladding light strippers consisting of $400 \mu \mathrm{m}$ diameter core-only fiber that was stripped and recoated in high refractive index UV adhesive $(n=1.52)$ over different length and with different fiber bending radii. The transmission through these devices was then measured with a goniometer. The result for a straight CLS with different length of UV adhesive is shown in Figure 11, where we have plotted the loss for light integrated from zero to the NA given on the x-axis (i.e., the light below 0.1 NA experiences $5 \mathrm{~dB}$ average loss for $2 \mathrm{~cm}$ of $\mathrm{UV}$ adhesive, for example).

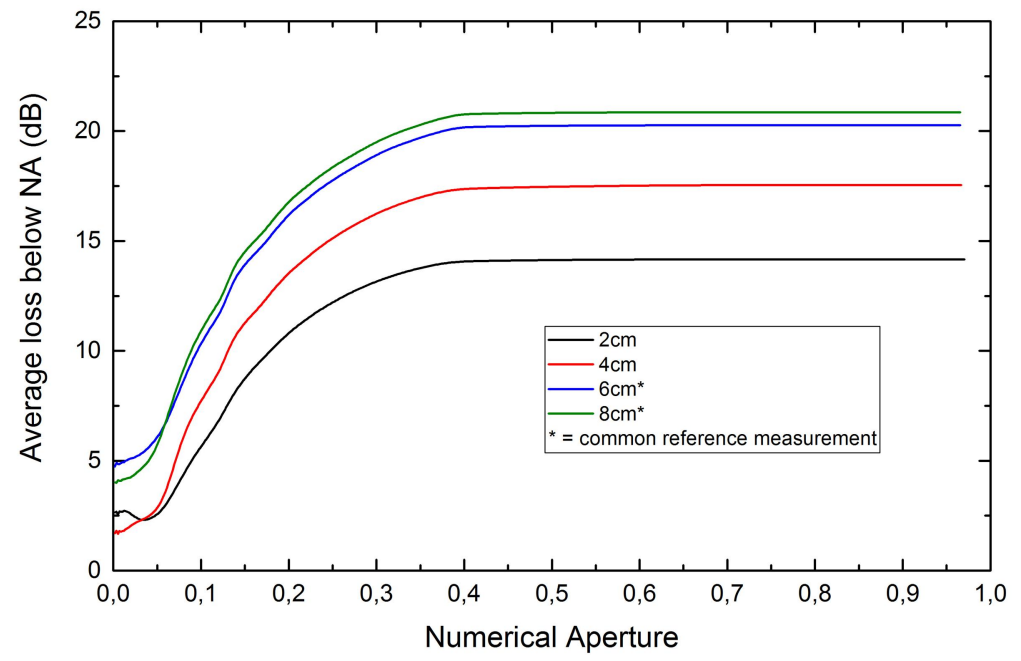

Figure 11: Loss of CLS with different lengths of UV adhesive integrated up to the NA plotted on the x-Axis.

It can be easily seen that low NA light experiences lower loss due to the CLS. This also causes the apparent saturationlike effect of the efficiency with respect to the device length: after a certain length, only low NA light is left in the fiber, which is in turn only stripped with low efficiency.

The efficiency for low NA light can be easily increased by bending the fiber in the CLS structure. Figure 12 shows the same accumulated average loss as Figure 11, but for a fixed length of $4 \mathrm{~cm}$ of UV adhesive with the fiber inside the adhesive bent at different bending radii. It can be seen that the efficiency for low NA light can be increased by more than an order of magnitude, from $\sim 2 \mathrm{~dB}$ to $15 \mathrm{~dB}$, by bending the fiber to $7 \mathrm{~cm}$ radius. While this may not be an option for grooved or etched cladding light strippers due to issues with mechanical stability, it provides an easy way to improve glued or coated CLSs that can be employed to remove remaining low NA light subsequent to high power CLS. 


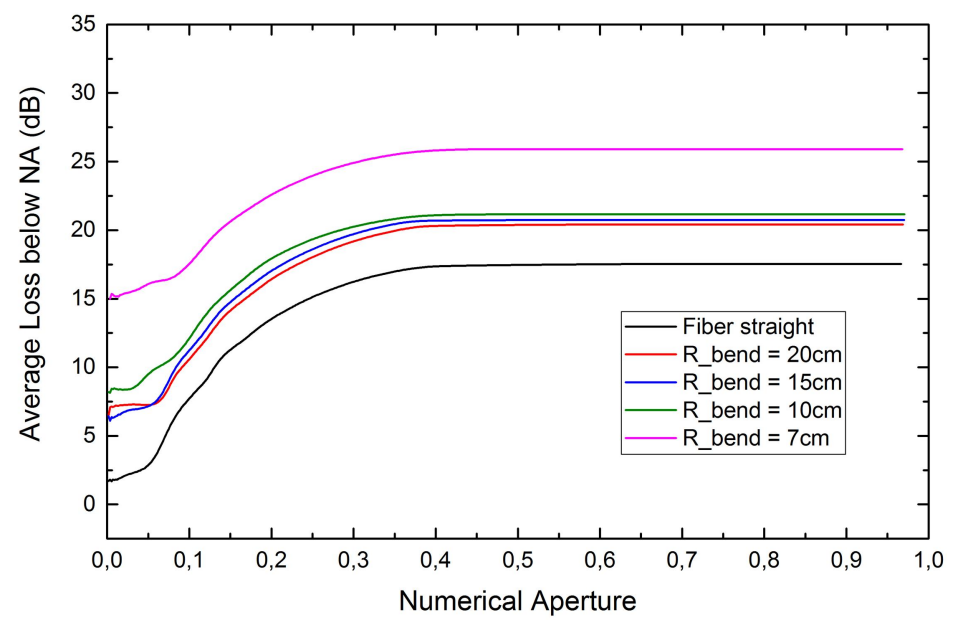

Figure 12: Loss of CLS ( $4 \mathrm{~cm}$ length) for different bending radii of the fiber inside the CLS.

\section{ACKNOWLEDGEMENT}

This work was supported by EURAMET under the EMPIR Program (Project PhotInd 14IND13).

\section{REFERENCES}

[1] A. Kliner et al., Proc. SPIE 8616, MOEMS and Miniaturized Systems XII, 86160N (March 13, 2013))

[2] Keiron Boyd, Nikita Simakov, Alexander Hemming, Jae Daniel, Robert Swain, Eric Mies, Simon Rees, W. Andrew Clarkson, and John Haub, "CO2 laser-fabricated cladding light strippers for high-power fiber lasers and amplifiers," Appl. Opt. 55, 2915-2920 (2016)

[3] S. Boehme, K. Hirte, S. Fabian, Ch. Hupel, T. Schreiber, R. Eberhardt, A. Tünnermann, "CO $2-l a s e r-b a s e d$ coating process for high power fiber application," Proc. SPIE 8968, Laser-based Micro- and Nanoprocessing VIII, 89680Z (6 March 2014); doi: 10.1117/12.2036357

[4] P. Yan et al., "Kilowatt-level cladding light stripper for high-power fiber laser," Appl. Opt. 56, 1935-1939 (2017). 